Anales de Literatura Hispanoamericana

ISSN-e: 1988-2351

\title{
Montevideo ou Une nouvelle Troie: dos finales en pugna
}

\author{
Alma Bolón ${ }^{1}$
}

Resumen. La obra de Alexandre Dumas, Une nouvelle Troie, tiene la particularidad de poseer dos finales, los cuales ofrecen dimensiones diferentes de la obra. Como ésta, durante su concepción y publicación, así como sus finales, fueron leídos en París y en Montevideo, es el asunto de este artículo.

Palabras clave: Alexandre Dumas; Un nouvelle Troie; París; Montevideo; censura.

\section{[en] Montevideo ou Une nouvelle Troie: two finals in dispute}

\begin{abstract}
Alexandre Dumas' Une nouvelle Troie has he peculiarity of having two endings, both of which offer different dimensions of the work. How it, during its conception and publication, as well as its endings, were read in Paris and Montevideo, is the subject of this articles.
\end{abstract}

Keywords: Alexandre Dumas; Un nouvelle Troie; Paris; Montevideo; censorship.

Sumario. 1. En Francia. 2. En el Río de la Plata.

Cómo citar: Bolón, A. (2021) Montevideo ou Une nouvelle Troie: dos finales en pugna, en Anales de Literatua Hispanoamericana $50,95-102$.

Como procuré exponerlo en anteriores oportunidades, el texto de Alexandre Dumas Montevideo ou Une nouvelle Troie ${ }^{2}$ es ficción, panfleto, ficción panfletaria, crónica, novela por encargo, y es novela histórica del tiempo apremiantemente presente, habida cuenta de que trata sobre la guerra, todavía en curso en el momento de su escritura y publicación, llevada adelante por el presidente uruguayo depuesto Manuel Oribe y su aliado el presidente argentino Juan Manuel de Rosas, contra el gobierno de Montevideo. Pero no solo desde lo genérico Montevideo ou Une nouvelle Troie presenta particularidades, sino que también desde su misma hechura. Por ejemplo, desde su inicio tuvo dos títulos, un título doble, dos autores, algunas variantes de importancia y un largo reposo, el que va desde su primera edición en 1850 a la segunda, en 2007 (Bolón, 2017).

En lo que sigue, retomaré la particularidad de sus dos finales, que dan dos extensiones diferentes a la obra, y buscaré concentrarme en la situación de la censura en París y en Montevideo, en aquellos meses en que la obra es concebida y publicada.

Antes, vale recordar nuestro punto de partida: algunos ejemplares de Montevideo ou Une nouvelle Troie constan de 167 páginas y otros de 174 (luego hablaré del caso particular que se da en los ejemplares que pude ver en Buenos Aires). Esta rareza queda patentemente documentada en el Manuel de l'amateur de livres du XIX siècle, 1801-1893, recopilación de Georges Vicaire publicada entre 1894 y 1920, en la que se proporcionan todos los datos editoriales de Montevideo ou Une nouvelle Troie, y se declaran 167 páginas, cifra tachada y corregida a mano por la de 174, al menos en el ejemplar del Manuel de l'amateur consultado, perteneciente a la BN de Francia (Vicaire, 1894-1920: 388$)^{3}$.

\footnotetext{
${ }^{1}$ Universidad de la República. Uruguay.

Email: abolon@vera.com.uy

${ }^{2}$ Alexandre Dumas. Montevideo ou Une nouvelle Troie (1850). Paris: Napoléon Chaix; reeditado por Amis d'Alexandre Dumas, Paris, 2007.

${ }^{3}$ Entrada "Dumas".
} 
En cuanto a la distribución geopolítica de estas versiones, se ha sostenido, tal como lo hace JacquesAndré Duprey (2007) que a Uruguay llegaron principalmente ejemplares con la versión larga de 174 páginas. La hipótesis es plausible y abundé en ella $(2017: 17,18)$, si bien no debe desconocerse la corrección a mano que presenta el Manuel de l'amateur de livres du XIX siècle; de igual modo no debe desconocerse el otro ejemplar, además de los dos pertenecientes a la BNf (ambos de 167 páginas), poseído por una biblioteca parisina pública, me refiero al ejemplar de 174 páginas del Fonds Lovenjoul de la biblioteca del Institut de France; tampoco deben desconocerse los ejemplares en venta en librerías de bibliófilos o rematados en París en Drout, a menudo también de 174 páginas.

Agrego ahora otro detalle elocuente: de los tres ejemplares que se encuentran en Buenos Aires, uno consta de 167 páginas y figura en el catálogo del Colegio Nacional de esta ciudad; los otros dos, que pude consultar en la Biblioteca Nacional, constan de 168, porque sus antiguos propietarios realizaron artesanalmente el trabajo de cercenar la obra de sus, en este caso seis, páginas finales. Sencillamente, estos lectores cortaron el final de la historia, cortando las tres hojas últimas, que poco debían de placerles ${ }^{4}$.

En efecto, en esas páginas ausentes en algunos ejemplares, Inglaterra y Austria son pintadas como regímenes opresores y enemigos de Francia, mientras que Italia y Hungría -y Montevideo, claro- son puestos como ejemplos de pueblos que luchan por la libertad. La versión más breve de Montevideo ou Une nouvelle Troie termina dramáticamente, ya que el narrador pregunta si la "civilisation" (los defensores de Montevideo) será socorrida por la "civilisation" a la que están pidiendo auxilio ("l’Europe") o si será abandonada a "la barbarie" (los atacantes de Montevideo, es decir, el presidente argentino Juan Manuel de Rosas y el uruguayo Manuel Oribe). Finalizando entonces el texto, el narrador expresa el temor de que el último grito lanzado por los sitiados a través de su propia voz sea inútil y se pierda: "Les abandonnera-t-on à la barbarie? Et le dernier cri qu'ils poussent par ma voix será-t-il un cri inutile et perdu?"

En las versiones largas, las de 174 páginas, Alexandre Dumas justamente responde esta pregunta: el grito -ese "grito postrero" que los sitiados lanzan a través de su voz de novelista célebre- será tan inútil y tan perdido como el lanzado por Francia a favor de los italianos y de los húngaros. El recuento de las relaciones mantenidas por Francia con Inglaterra y con Austria a lo largo de los últimos cuatro siglos solo muestra, escribe Dumas, que ambas son "nos ennemies mortelles". Solo la pequeñez de los pequeños hombres ("les petits hommes") que gobiernan un "gran pueblo" ("grand peuple") impide pelear por "la cause sainte de la liberté", pero algún día llegará la reparación, por lo que en este segundo final del que está provisto un número indeterminado de ejemplares de esa edición, Dumas se dirige directamente a los pueblos que, a sus ojos, encarnan la lucha por la libertad: "Patience Italiens, patience Hongrois, patience Montevidéens, un jour viendra où tout un peuple de républicains vous dira: $-[\ldots]$ oubliez notre intervention à Nésib, notre présence à Rome, notre absence à Montevideo. En attendant Kossuth, en attendant Mazzini, en attendant Suárez, celui qui écrit ses lignes à votre honneur vous demande, pour toute récompense, sa place de citoyen dans vos républiques à venir".

Patentemente, si en el título Dumas presentaba aquella guerra en clave épica y mitológica -una nueva Troya se había armado en un paraje lejano: Montevideo-, en este final cercenado, el conflicto rioplatense encuentra su lugar en una historia política contemporánea, en el conflicto planetario entre la grandeza de los pueblos y la pequeñez de sus gobernantes, en el combate entre los estados despóticos y los pueblos republicanos. De igual modo, la Troya rioplatense pasa a inscribirse en el mapa del desengaño que entonces experimentan los republicanos que, como Alexandre Dumas o como Victor Hugo, habían apoyado a LouisNapoléon Bonaparte luego de la revolución de febrero de 1848, apoyo que, entre los últimos meses de 1849 y enero o febrero de 1850 (dentro de ese lapso se escribió Montevideo ou Une nouvelle Troie) se encontraba francamente carcomido, dadas las políticas llevadas adelante por el príncipe todavía presidente pero ya en camino imperioso, si no imperial.

Si la revolución de febrero de 1848 y la instauración de la II república habían encendido los corazones en ambos lados del mar (en Montevideo, el periódico Le Patriote Français ${ }^{5}$ publicitaba cotidianamente la venta en sus locales de la "Constitution de la République Française promulguée par l'Assemblée Nationale le 12 novembre 1848 ”), pronto habrá motivos para la decepción y para el temor. El final dumasiano libertario -

\footnotetext{
${ }^{4}$ Varias inscripciones en estos ejemplares dan a conocer algunas de las manos por las que pasaron.

${ }^{5}$ Le Patriote Français es un periódico en lengua francesa que se publicó en Montevideo a lo largo de los siete años que duró el sitio a esta ciudad, entre 1843 y 1850.
} 
presente y ausente según los ejemplares- da cuenta de ese doble movimiento que sigue el novelista, experimentación de un desengaño y consecuente prudencia.

\section{En Francia}

En efecto, durante la Segunda República, en cuyos mediados se escribe Montevideo ou Une nouvelle Troie, se sucede una serie de leyes y decretos (11 de agosto de 1848, 27 de julio de 1849, 16 de julio de 1850) destinados a coartar el ejercicio de la libertad de prensa, que a lo largo de todo el siglo constituye "le cœur du combat politique" (Robert, 2012: 62). Por ejemplo, el decreto de agosto de 1848, pergeñado luego de las masacres obreras de junio de ese año -"Silence aux pauvres!” escribía entonces Lamennais (Robert, 2012: 61)- duplica la duración de la pena de cárcel y el monto de la multa a quien atentase contra la paz pública mediante "provocation à la haine entre les différentes classes de la société". En el correr de esos años vuelve a instaurarse "le cautionnement", es decir, grandes cantidades de dinero que los periódicos deben entregar por adelantado, para pagar posibles condenas, así como también se amplían los casos que configuran delitos por vía de prensa: ataques a la Asamblea Nacional, a la libertad de cultos, a las instituciones republicanas, a la propiedad, a la familia; en julio de 1849, también se instaura la figura de "ofensa al Presidente de la República" y un año más tarde se restablece el "timbre", impuesto fiscal que debía pagar la prensa que publicaba folletines (debía pagarse un céntimo por ejemplar).

Alexandre Dumas, a la sazón editor y único autor de Le Mois (marzo de 1848-febrero de 1850), en las páginas de este periódico va dejando traslucir su decepción, a medida que los anhelos de república fraterna van encontrando un régimen autoritario y burgués. Aunque en el otoño europeo de 1849 Dumas haya vuelto a apoyar a Louis-Napoléon, ya los últimos números de Le Mois anuncian "obsessionnellement la menace du coup d'État" (Mombert, 2012: 1129). En el último número, Dumas se dirige directamente a Louis-Napoléon: "Non, la France a confiance en vous, cette confiance será justifiée. Non, il n'y aura pas de coup d'État", tal vez con la esperanza de ser oído y, como artista popular, ser instituido interlocutor legítimo del poder. Nada de esto sucederá y su periódico ya no volverá a ver la luz (Mombert, 2012: 1129).

Justamente, será en los dos últimos números de Le Mois, los correspondientes a enero y a febrero de 1850, que Alexandre Dumas publicará los dos primeros capítulos de Montevideo ou Une nouvelle Troie que saldrá de la imprenta de Napoléon Chaix en marzo o abril siguientes. La escritura de ese final agónico que pregunta por el destino del grito que lanzan, por su intermedio, los montevideanos sitiados coincide con el final de su empresa periodística, nacida en la esperanza de la revolución de febrero de 1848.

En cuanto a la censura de los textos dramáticos que entonces se llevaban a las tablas, ésta estuvo muy activa a lo largo de todo el siglo XIX e inclusive más allá de la liberalización de 1881, puesto que la censura previa a la que estaban sometidos los textos dramáticos se extendió hasta 1906, no obstante se haya interrumpido por breve lapso entre 1830 y 1835 (es decir, durante los primeros cinco años de la Monarquía de Julio), y luego volvió a interrumpirse de febrero de 1848 a 30 de julio de 1850 (Krakovitch). A lo largo de todo el siglo XIX, salvo durante escasos ocho años, los autores no pudieron prescindir de ir a someter sus obras de teatro a la oficina del censor. Y, como revelan los archivos franceses que guardan traza de la censura, ésta estuvo bien activa hasta los primeros días de febrero de 1848. Así por ejemplo, hasta el 3 y 4 de febrero de 1848 se documentan trámites ante la oficina de censura de Beaux-Arts, más precisamente ocasionados por "Le château d'If", primera parte del Conde de Montecristo, para cuya puesta en escena en el Théâtre Historique (justamente perteneciente a Alexandre Dumas), se tramita su autorización por el ministerio del Interior (ANF, F21/995) y cuyas representaciones serán interrumpidas por la revolución, pocos días más tarde. También hay un informe del Ministère de l'Intérieur-Direction des Beaux-Arts-Bureau des Théâtres, en enero de 1848, correspondiente a la pieza "Thérèse Lambert" y para "Hortense de Blangy", de la que consta un borrador y una pasada en limpio, que acompañan la conclusión del censor: "Nous proposons l'autorisation, à la charge des modifications opérées, faites sur le manuscrit" (ANF, F21/974). Luego, durante todo el año 1849, no hay más registros de censura, hasta que esta vuelve en 1850, en donde hay por lo menos trece expedientes de varias páginas cada uno, que abarcan desde agosto, retorno de la censura teatral, a octubre. Se prohíben entonces "Le Juif errant" y "Les Mystères de Paris", las novelas que Eugène Sue, al igual que Dumas, dramatizaba.

Veamos lo concienzudo de la labor de la censura, que para nada se efectúa al tuntún, sino luego de pormenorizados análisis textuales, con parlamentos de los textos subrayados, citados y referenciados en la obra dramática (por ejemplo, Acto Z, Escena W). El 19 de agosto de 1850, es decir, pocos meses después de que haya salido de la imprenta Montevideo ou Une nouvelle Troie, y, recuerdo, luego de haberse vuelto a instaurar la censura teatral, se somete al ministerio del Interior "Le bonhomme Jacques", drama en cinco actos; esto da lugar a un informe de cuatro carillas, en letra apretada, firmado por cuatro censores, que 
concluyen: "Le prologue ou 1er acte de cette pièce a pour objet un crime qui, de toute façon, devrait être banni du théâtre, mais dont la mise en action, comme ici, devient une monstruosité scénique" (ANF, F21/976).

El año siguiente, en septiembre de 1851, ya en los umbrales del golpe de Estado, un informe resume "La peau de chagrin", concluyendo: "Nous proposons l'autorisation de ce drame, à la charge des nombreuses modifications de détail que les auteurs y ont apporté sur notre demande".

Esto suponía, claramente, desde antes del golpe de Estado, una vuelta a la Monarquía de Julio, que había intentado sobrevivir mediante el control de la prensa y de la ficción teatral. Veamos dos informes de la censura a propósito de obras de Alexandre Dumas: el primero corresponde a la Monarquía de Julio y el segundo corresponde a lo que todavía es, formalmente, la Segunda República. En enero de 1847, Dumas inaugura el Théâtre historique con "La reine Margot". Los censores de la Direction des Beaux-Arts del Ministère de l'intérieur escriben: "Nous avons vu avec regret le choix d'un pareil sujet, pour l'inauguration du Théâtre historique, malgré les nombreuses modifications que nous nous proposons de demander nous craignons qu'il ne soit pas son sujet même qu'on ne peut changer de nature à porter atteinte au respect dû à la religion et à la royauté, l'auteur y présentant en effet des scènes nombreuses d'empoisonnement et de débauche à la cour de France. Nous pensons toutefois qu'il y aurait plus d'inconvénient à en empêcher la représentation qu'à la tolérer $[\ldots]$ nous mettons sous les yeux les principaux passages qui nous ont parus fâcheux" (ANF, F21).

Igualmente, meses más tarde, en septiembre de 1847, Dumas somete a los censores la primera parte de "Le comte de Monte-Christo" (sic) y los censores dicen que antes de opinar sobre esa primera parte, quieren conocer la segunda... un post scriptum de enero de 1848 concluye:

notre incertitude est maintenant finie et la direction du Thêâtre historique nous a déclaré que le drame de Monte-Christo (sic) ne s'étenderait pas au-delà de la première partie qui serait jouée en deux soirées [...] sur notre demande l'auteur a introduit dans ce drame deux modifications importantes. Faria n'est plus un prêtre et la qualification d'abbé qu'on lui donne encore une ou deux fois seulement est expliquée par son savoir et parce que dans sa jeunesse, il s'était préparé à l'état ecclésiastique. Villefort n'est plus un substitut du procureur du roi, ou laisse dans l'indécision sa qualité, qui peut être celle de secrétaire du parquet ou d'avocat ou de témoin dans l'affaire de Bertuccio. Mme de Margonne ne paraît plus en scène. (ANF, F21)

Estos registros muestran la lectura profunda que hacen los censores, acusando recibo de los golpes que el autor procura asestar con sus obras; también muestran la disposición de éste para negociar el texto, sacrificando lo necesario. Igualmente, estos registros permiten imaginar cierta forma de hastío por parte de Dumas, obligado a esos regateos, y la consiguiente esperanza que a fines de febrero de 1848 le despierta la caída de la monarquía y la desaparición de la censura. Cuando ésta vuelva, e irá volviendo paulatinamente, el proceder de la censura será similar, si no es más encarnizado.

Así, en marzo de 1851, a un año de la publicación de Montevideo ou Une nouvelle Troie, todavía, formalmente, Segunda República, vuelven a encontrarse informes de censores sobre "Le comte de Morcerf" (segunda parte de Monte-Cristo). Los censores resumen la obra y luego concluyen:

Toutes ces scènes sont traitées avec convenance. Néanmoins, la situation d'un pair de France placé dans des pareilles circonstances a appelé toute notre attention. Bien que la pairie ne fasse plus partie des institutions actuelles, cette élite des illustrations nationales a conservé de puissantes sympathies. Beaucoup de ses membres occupent dans l'opinion et dans l'assemblée une place considérable. D'ailleurs les pouvoirs publics, qui représentent l'autorité, ont droit au respect, même après leur chute. Dans cette position, nous regrettons qu'un personnage comme Morcerf soit revêtu de la condition de pair de France. [...] En conséquence, nous proposons l'autorisation. Toutefois nous croyons devoir appeler l'attention de Monsieur le Ministre sur une question aussi délicate et la soumettre à sa haute appréciation.

Un mes más tarde, los censores se expiden sobre "Villefort", drama en cinco actos. Luego del resumen apretado, lo dan por conocido y concluyen:

Plusieurs choses dans ce drame nous ont paru présenter de graves inconvénients soit dans les situations, soit dans les détails. La plus importante de toutes est le personnage de Villefort, magistrat coupable à la fois d'adultère, de soustraction d'enfant et d'abus exorbitant de pouvoir dans l'exercice de ses fonctions. Un procureur général siégeant en cour d'assises, achevant son réquisitoire [...] Nous avons donc été unanimement d'avis que ce tableau (le $12^{\mathrm{e}}$ ) était radicalement impossible dans son état actuel. Il nous a également paru dans les convenances que l'acte arbitraire sur lequel se fondent les vengeances de Monte- 
Cristo ne fut pas le fait d'un magistrat mais de toute autre personnage n'appartenant pas au parquet, comme serait par exemple un commissaire extraordinaire envoyé en 1825 à Marseille; et nous avons été d'autant plus confirmés dans ce sentiment que la pièce du comte de Monte-Cristo représentée en 1849 sur le Théâtre historique n' a été autorisée qu'à la condition expresse que Villefort ne serait plus substitut du procureur du Roi et qu'on laisserait sa qualité dans l'indécision. [...] Par la suite, M. Alexandre Dumas, auteur de la pièce, a proposé dans un écrit signé de lui et ci-joint, au lieu d'une séance de cour d'assises et d'un procureurgénéral, de faire passer la scène dans le cabinet de $\mathrm{M}$. de Villefort, qui ne serait plus qu'un simple magistrat. [...] Ce changement capital ôtant au tableau l'effet dangereux [...] l'adresse et l'habilité éprouvées de l'auteur pourraient rendre ce nouveau tableau admissible. (ANF, F21)

El expediente se continúa en varias fojas más de posterior fecha, que incluyen la final autorización, obtenida gracias a que el autor rehízo totalmente el cuadro $12^{\mathrm{e}}$ y la escena en la cour d'assises fue trasladada a otro marco menos comprometedor para el poder.

En este marco tenso con la censura, cuando publica Montevideo ou Une nouvelle Troie, Alexandre Dumas parece haber cortado por lo sano: una versión cercenada será declarada y entregada a la Bibliothèque Nationale, y acto seguido se agregarán los plomos en que Dumas deja lugar no ya el tiempo apremiantemente presente de la Troya rioplatense, sino al tiempo apremiante europeo, el que Inglaterra y Austria imponían, el tiempo contemporáneo de las luchas de los italianos y de los húngaros.

En efecto, en esos mismos días en que Alexandre Dumas, a instancias del montevideano Melchor Pacheco y Obes, se interiorizaba del conflicto rioplatense y empezaba a concebirse Montevideo ou Une nouvelle Troie, Victor Hugo denunciaba en la Assemblée nationale la derrota de los italianos en manos de los austríacos y, sobre todo, las exacciones de estos, así como cuestionaba el papel desempeñado por la intervención en Italia del ejército francés, que él mismo había votado y que ahora le llevaba a preguntarse qué estaban haciendo allí los franceses, si no era oficiando de aliados de austríacos y del papado.

En cuanto a la dumasiana diatriba anti Inglaterra, nada más inoportuna, ya que "l'entente cordiale", expresión empleada por Guizot en 1830 para nombrar la cooperación y el entendimiento diplomático entre Francia e Inglaterra, está revigorizándose en los años de la Segunda República, que puso a su cabeza a un príncipe-presidente que había pasado su largo exilio en Inglaterra y que, una vez que haya consumado el golpe del 2 de diciembre de 1851, una vez que haya consumado su propio 18 Brumario, será caracterizado de "agente inglés", por precisamente Karl Marx.

\section{En el Río de la Plata}

Cuando prontamente llegan a Montevideo las primeras versiones de la novela de Alexandre Dumas, este alegato bélico también resulta muy inoportuno, al menos para quienes están buscando terminar con la guerra, por convicción y/o por escasa estima a las partes beligerantes. Entre estos se encuentra Devoize, cónsul general de Francia (entonces no hay embajada francesa).

En agosto de 1848, Antoine Devoize, cónsul general de Francia en Montevideo, había transmitido a su ministerio en París el "júbilo" ("enthousiasme") expresado por Manuel Herrera y Obes ante la proclamación de la Segunda República ${ }^{6}$. De hecho, desde el 12 de junio de 1848, estaba vigente el subsidio que el gobierno de Francia otorgaba a los defensores de Montevideo y cuyo pago el cónsul Devoize no dudará en dosificar y demorar para presionar al gobierno montevideano, con el que se encuentra en perenne malestar.

La correspondencia de Devoize sobre la situación montevideana contrasta con el cuadro dumasiano de una Montevideo "dernier boulevard de la civilisation". Por ejemplo: "si, le jour, vous voyez, sur la route de ces belles quintas, des groupes des femmes en amazones, de cavaliers en habit de cheval; si, le soir, à travers les fenêtres ouvertes, et versant dans les rues des torrents de lumière et d'harmonie, vous entendez les chants des pianos ou les plaintes de la harpe, les trilles pétillantes des quadrilles ou les notes plaintives des romances, c'est que vous êtes à Montevideo, la vice-reine de ce grand fleuve d'argent dont Buenos Aires prétend être la reine", estampa en los primeros párrafos de su obra Dumas.

En el mismo año, Devoize había escrito a su gobierno: 
À partir de 1847, les actes d'extorsions et de violences deviennent moins fréquents de la part des autorités Orientales et de la garnison. Rivera, Pacheco et Garibaldi avaient d'ailleurs été écartés de la scène politique. [...]. Les désordres, les injustices, les spoliations n'affectent plus des formes aussi violentes que par le passé, mais on trouve toujours pour leur assurer l'impunité quelque fonctionnaire qui à la faveur d'une enquête mensongère ou d'une formalité trompeuse, parvient à leur donner des apparences légales; l'affaire dans laquelle le droit est la plus incontestable devient alors interminable, et la partie lésée préfère subir le dommage plutôt que de recourir à une façon de Tribunal civil que fonctionne parfois à Montevideo et qui n'inspire et ne mérite aucune confiance. J'ai déjà dit que les vols et les assassinats ne donnaient pas même lieu à un simulacre d'instruction de la part du Tribunal du Crime $[\ldots]^{7}$

La versión de Devoize no solo diverge con el Montevideo dumasiano en cuanto a que las "espoliaciones", los "asesinatos" y las "injusticias impunes" ocupan el lugar de los "torrentes de luz y de armonía" y de los "trinos de los pianos y de las harpas". También, Devoize atribuye al alejamiento de Pacheco y Obes (junto con los de Rivera y Garibaldi) un leve simulacro de mejoría en la ciudad, mientras que Dumas hace de Pacheco y Obes el héroe -el Marius- de la gesta troyana rioplatense. La prédica anti Pacheco y Obes que lleva adelante Devoize se vuelve mucho más directa en la continuación de su informe, cuando relata acontecimientos acaecidos siete años atrás, es decir, cuando faltaba todavía bastante para que Montevideo fuera el destino diplomático de este informante: Devoize no solo desea transmitir a sus superiores un informe de actualidad, sino también parte de lo que considera el prontuario de Melchor Pacheco y Obes:

En efecto, en "1843, le gouvernement de cette ville ayant besoin d'argent", decretó que el dinero fuera declarado: "sous des peines très sévères en cas d'infraction. Ces déclarations ayant été faites, des soldats sous les ordres du Général Pacheco (actuellement envoyé extraordinaire à Paris) se rendirent chez les dépositaires et s'emparèrent de ces dépôts pour les besoins de la patrie. Les mêmes violences furent commises par ce même général et pour les mêmes motifs sur des Espagnols qui n’ayant pas de dépôt à lui remettre durent lui livrer leur argent, leurs objets précieux, leur argenterie pour le Gouvernement." 8

Unas semanas antes, Devoize también se había referido a Pacheco, y más precisamente, a Montevideo ou Une nouvelle Troie, novela que, hasta ese entonces, venía siendo publicada por, entre otros, el periódico montevideano Le Patriote Français. Escribe el cónsul Devoize a su ministro:

Le Gal Pacheco, de nouveau chargé d'une mission extraordinaire auprès du gouvernement de la République, partira au premier jour sur un bâtiment du commerce Italien destiné pour Gibraltar. Cette fois comme la première, son gouvernement a voulu l'éloigner de Montevideo. Dans l'inutilité des efforts que des partisans ont faits, à son retour, pour exciter l'enthousiasme de la population, M. Pacheco a pu voir combien leur nombre était restreint. D'autres ambitions s'étaient produites auxquelles la sienne faisait ombrage. Le Roman de la Nouvelle Troie dont il a fourni lui-même les éléments a jeté sur lui, par l'exagération impudente dont il y est parlé de sa personne et des principaux personnages de Montevideo, un ridicule et une défaveur qui se sont ajoutés à toutes les méfiances que sa conduite précédente avait excitées. Pendant les 3 mois qu'il vient de passer ici, l'opinion publique s'y est prononcée contre sa rentrée au Ministère, l'armée a montré peu d'empressement à accepter son commandement, et il s'est, en définitive, tenu dans une assez grande réserve. ${ }^{9}$

Por cierto, en este relato, Devoize refiere a las autoridades en París los datos que recoge en los ámbitos del gobierno de la Defensa, tal como lo había hecho en carta anterior, también denigrante hacia Melchor Pacheco, identificado como el principal "partidario de la guerra", junto con los "refugiados argentinos" y "toda la prensa montevideana", todos ellos prontos para hacer una "résistance d'autant plus énergique" en cuanto el general Pacheco “a été accueilli par ses adhérents avec un enthousiasme d'autant plus grand qu'il avait fait plus de bruit à Paris à en juger par ses publications et ses dépêches répandues ici en grand nombre dans le public" $" 10$.

En la carta del 7 de agosto, el cónsul se refiere explícitamente a "le Roman de la Nouvelle Troie" omitiendo cualquier mención a Alexandre Dumas, notoriamente célebre entonces en Francia y en

\footnotetext{
${ }^{7}$ Carta de A. Devoize del 17/IX/1850, Archives diplomatiques de Nantes.

${ }^{8}$ Idem. Devoize, en la misma carta, también relata exacciones de las tropas de Manuel Oribe, y manifiesta conocer "la difficulté de rencontrer des hommes impartiaux, éclairés et d'une position indépendante qui consentent à accepter une semblable mission au milieu de tous les désordres des mœurs et des institutions du Pays et en présence de Gouvernements tels que ceux de la Plata".

${ }^{9}$ Carta del 7/VIII/1850.

${ }^{10}$ Carta del 25/VI/1850, escrita la semana siguiente del retorno de Pacheco a Montevideo, que esta carta precisamente fecha en el día 16 de junio.
} 
Montevideo. En ésta del 25 de junio, brilla por su ausencia cualquier referencia a la novela dumasiana, si no es bajo el vago "ses publications", es decir las publicaciones de Melchor Pacheco, "répandues ici en grand nombre dans le public"; esto es llamativo, porque para esas fechas Melchor Pacheco y Obes ya había regresado a Montevideo con ejemplares de la novela recién salida de la imprenta parisina que, por otra parte, ya estaba siendo difundida en la prensa montevideana, desde un mes atrás, a partir de los adelantos publicados por Le Mois ${ }^{11}$. Sin duda, por poco entusiasta que fuera el cónsul Devoize de la literatura dumasiana, el hecho de que uno de sus principales detestados montevideanos hubiera conseguido la simpatía del famoso escritor parisino no debía de serle fácil de tramitar ${ }^{12}$.

De hecho, José Ellauri, representante en París del gobierno de la Defensa de Montevideo, envía al gobierno francés una extensa carta con las quejas que el gobierno montevideano tiene contra el cónsul Devoize "systématiquement hostile à mon gouvernement et à toute la population de Montevideo, soit nationale, soit étrangère". En esta carta de denuncia contra Devoize, Ellauri no duda en hacer suyas algunas formulaciones de Dumas: "les agents français dans la Plata semblent prendre à tâche d'affaiblir et de discréditer par tous les moyens possibles l'héroïque défense de Montevideo, unique boulevard aujourd'hui dans ces contrées de la liberté, de l'ordre, de la civilisation et des lumières, contre la tyrannie la plus barbare et la plus effrennée". ${ }^{13}$ El canciller francés responderá con una defensa cerrada de Devoize, descalificando las quejas de Ellauri, llamándolas "accusations vagues" y atacando al diario montevideano en francés "Le Patriote Français", acusándolo de participar en el reclutamiento por las legiones extranjeras (francesa, vasca, italiana) de buena parte de los desertores (franceses) de la escuadra (francesa) ${ }^{14}$.

Contemporáneo de este correo, hay otro en que París se dirige a su cónsul Devoize para anunciarle que "l'arrangement qui se négociait entre la France et l'Angleterre vient d'être conclu sur les bases que nous désirions"; el acuerdo, en este caso, tiene que ver con la situación en Grecia... 15 .

Es entonces en este campo atravesado por múltiples conflictos que recortan, atraviesan y solapan nacionalidades, procedencias y enemistades seculares, que se escribe y se difunde Montevideo ou Une nouvelle Troie, obra en que aparecen representados solo dos grandes contendientes, mientras su autor apela a Francia para que, sin ambages, se sume a uno de los dos bandos. Como fue dicho, si hay pocas huellas de la promoción que recibió el libro traído a Montevideo, Le Patriote Français en dos oportunidades inició su publicación como folletín. Me he referido en anterior oportunidad a la primera interrupción, retomo ahora, con nuevos elementos, la segunda y última, la que deja definitivamente inconclusa la publicación del texto dumasiano en francés en la prensa montevideana.

En efecto, el 3 de agosto de 1850, Manuel Herrera y Obes firma un decreto de suspensión de Le Patriote Français por el término de dos meses, y así lo dice: "por obra exclusiva de los deberes con que el Gobierno se considera para consigo mismo y para con los agentes de las Naciones Amigas, que existen en la República bajo la protección", sanciona al periódico por haber publicado en fecha reciente el artículo "Simple rapprochement", que censura a Le Prédour en "su doble carácter de negociador y Agente Público del Gobierno Francés", al emplear "términos y conceptos que el Gobierno no puede tolerar porque importan ofensas e injurias que tiene el deber de reprimir en el interés de su honor, de la justicia y del respeto que merecen sus mandatos conocidos de los Redactores de ese Diario y violados en esa publicación"16.

El artículo invocado por el gobierno de la defensa de Montevideo para clausurar por dos meses Le Patriote Français consiste en dos proclamas publicadas en primera página, ambas de autoría de Le Prédour; en una de ellas, figura el texto "Proclamation aux Légions", fechado el 20 de mayo de 1849, en la otra figura "Proclamation à l'Escadre", con fecha del 23 de julio de 1850. El contraste entre lo dicho a unos y a otros es llamativo, y Le Patriote Français concluye el cotejo diciendo que Le Prédour "prolonge inutilement les souffrances d'une alliée fidèle et dévouée au profit de la politique anglaise et d'un béligérant obstiné dont la mauvaise foi est passée en proverbe" 17 .

Este "simple acercamiento" de las dos proclamas de Le Prédour permiten al Patriote Français denunciar el tratado que este militar y diplomático francés estaba pergeñando para terminar la guerra, y justamente con

\footnotetext{
${ }^{11}$ Cf. Alma Bolón "Sobre una página manuscrita de Montevideo ou Une nouvelle Troie, ..."

${ }^{12}$ Leonardo Rivero Torielli, quien también leyó concienzudamente la correspondencia del cónsul Devoize, me confirma en comunicación personal que esta es la única referencia explícita que el diplomático hace del texto dumasiano sobre Montevideo.

${ }^{13}$ Carta del 3/VIII/1850.

${ }^{14}$ Copia de carta del ministro general de la Hitte, en respuesta a la carta de José Ellauri, 16/VIII/1850.

${ }_{15}^{15}$ Post Scriptum fechado el 21/VI/1850 a carta del 15/VI/1850.

${ }^{16}$ Suspensión du Journal Le Patriote Français, Manuel Herrera y Obes a Devoize, 3/VIII/1850; Archivos de Nantes.

17 “Simple rapprochement", Le Patriote Français, 31 de julio de 1850.
} 
esto se denuncia la supeditación de Francia a Inglaterra, denuncia realizada por los propios ciudadanos franceses emigrados a Montevideo y partícipes de su defensa. Paradójicamente, será el gobierno de la Defensa, quien clausure el periódico por dos meses.

Puede también conjeturarse que, a la razón invocada por Manuel Herrera y Obes para sancionar al Patriote Français, se suma otra. En efecto, como decíamos, este periódico había reiniciado la publicación, como folletín, de Montevideo ou Une nouvelle Troie, adoptando la versión del libro impreso traído por Melchor Pacheco y Obes. En el mismo número que se publica "Simple rapprochement", salen las últimas páginas del capítulo III del texto dumasiano, capítulo que se concluye con la ejecución por parte de las tropas rosistas de Camila O'Gorman y del bebé que lleva en su vientre, episodio que da lugar a las siguientes palabras del narrador: "Maintenant, comment se fait-il que la France se fasse des ennemis comme Garibaldi, se fasse des amis comme Rosas? C'est que les amis et les ennemis de la France lui sont imposées par l'Angleterre".

Aunque desde la ficcionalización dumasiana y desde el cotejo textual periodístico se está hablando casi que con una única voz, el gobierno de Montevideo solo mencionará el texto periodístico, como motivo de la censura. Cuando el periódico vuelva a aparecer dos meses después, su redactor responsable ya no será Arsène Isabelle y también se habrá modificado la impronta que el ex canciller del consulado francés en Montevideo había dado al Patriote Français, desde que asumiese poco tiempo antes su dirección. Pero, sobre todo, cuando el periódico vuelva a aparecer el 29 de septiembre siguiente, ya no se retomará la publicación en folletín de Montevideo ou Une nouvelle Troie, quedando así en suspenso los tres últimos capítulos y ejerciéndose así una especie de complemento a la autocensura que, en París y algunos meses atrás, se había autoimpuesto Alexandre Dumas, al tomar la precaución de publicar y dar difusión un texto menos condenatorio de Inglaterra y, sobre todo, menos condenatorio de la supeditada política diplomática francesa.

\section{Referencias bibliográficas}

Archives Nationales de France (ANF).

Bolón Pedretti, Alma (2017), "Sobre una página manuscrita de Montevideo ou Une nouvelle Troie, sobre algunas variantes". Lo que los archivos cuentan. Montevideo: Biblioteca Nacional, 2017, págs. 11-41.

Dumas, Alexandre (1850a). Montevideo ou Une Nouvelle Troie. Paris: Napoléon Chaix, 1850, 167 páginas.

Dumas, Alexandre (1850b). Montevideo ou Une Nouvelle Troie. Paris: Napoléon Chaix, 1850, 174 páginas.

Krakovitch, Odile (s.f.), "Histoire de la censure du théâtre à Paris au XIX ${ }^{e}$ siècle", en Censure des répertoires parisiens des grands théâtres parisiens (1835-1906).

Le Patriote Français. Montevideo, 1843 - 1850.

Mombert, Sarah (2012), “Alexandre Dumas (1802-1870)”, en Alain Vaillant, Dominique Kalifa, Marie-Ėve Thérenty, Philippe Régnier (dirs.). La civilisation du journal. Histoire culturelle et littéraire de la presse dans le XIXe siècle. Paris: Nouveau Monde Ėditions, págs. 1125-1133.

Robert, Vincent (2012), “Lois, censure et liberté”, en Alain Vaillant, Dominique Kalifa, Marie-Ėve Thérenty, Philippe Régnier (dirs.). Paris: Nouveau Monde Ėditions, 2012.

Vicaire, Georges (1894-1920). Manuel de l'amateur de livres du XIX siècle, 1801-1893; 8 vol. in-8. Paris: A. Roquette, Tome III, pág. 388. 